\title{
Registration of Positron Emission Tomography (PET) Image and Functional Near Infrared Spectroscopy (NIRS) Data
}

\author{
Fairuz Binti Mohd Nasiri, 2, Asuka Kikuchi ${ }^{4}$, Shoichi Watanuki ${ }^{4}$, Masayasu Miyake3, Manabu \\ Tashiro ${ }^{4}$, Hiroshi Watabe ${ }^{3 *}$ \\ ${ }^{1}$ Graduate School of Biomedical Engineering, Tohoku University, Sendai, Japan. \\ 2 University Sultan Zainal Abidin, Kuala Terengganu, Malaysia. \\ ${ }^{3}$ Divisions of Radiation Protection and Safety Control, Cyclotron and Radioisotope Center, Tohoku \\ University, Sendai, Japan. \\ 4 Divisions of Cyclotron Nuclear Medicine, Cyclotron and Radioisotope Center, Tohoku University, Sendai, \\ Japan. \\ * Corresponding author. Tel.: +81-22-795-7803; email: watabe@cyric.tohoku.ac.jp \\ Manuscript submitted February 5, 2018; accepted July 12, 2018. \\ doi: 10.17706/ijbbb.2019.9.1.9-19
}

\begin{abstract}
Functional near infrared spectroscopy (NIRS) is the optical imaging that measures changes of blood hemodynamic while positron emission tomography (PET) measure physiological condition of the interest area. NIRS has high temporal resolution while PET image has better spatial resolution compared, however, NIRS measures hemodynamic changes in the cortex region but not in deeper brain structures. Therefore, this study is aimed to register the both image; the NIRS data on the PET image. The registration of NIRS on PET image was done on eleven subjects which undergoes NIRS and PET scan separately. To register the NIRS probe on the PET image, we did a transformation of NIRS probe coordinate to the PET coordinate based on Polaris marker position attached to the NIRS cap. The coordinate of these markers is obtained using the optical tracking system, Polaris. The resulting image forms PET and NIRS were visually aligned as well as the coordinate of the marker obtained during the PET acquisition. The probe was registered on the PET image using the in-house software. The registration done in this study is considered successful as we can view the NIRS activation area on PET image.
\end{abstract}

Key words: Functional near-infrared spectroscopy, PET-NIRS, multimodal image registration.

\section{Introduction}

In recent years, medical image registration has gained considerable attention to gather as much information as possible for the diagnosis of the disease. Calvin Maurer (1993) defined the image registration as the determination of one-to-one mapping between the coordinates in one space and those in another such that points in the two space that correspond to the same anatomical point are mapped to each other [1]. The goal of image registration is to align the images with respect to each other, match the corresponding images based on certain features to assist in the image fusion and to produce as output geometrical transformation that aligns corresponding points and can serve as input to a system further along in the image acquisition [1], [2].

Recently, there has been many modalities in radiology as well as nuclear medicine where each of them 
has their own purpose and advantages. To optimize the advantages of each modality, multimodal imaging is available to help a better diagnose. This lead to the multimodal image registration which is matching images of the same patient which acquired by different imaging technologies. Normally the registration image is in between integration of anatomical and functional image. Most common multimodal image registration is magnetic resonance imaging (MRI) and positron emission tomography (PET), PET and computed tomography (CT), single photon emission computed tomography (SPECT) and CT.

This paper focuses on registering the functional near-infrared spectroscopy (NIRS) on positron PET image. This is a multimodal image registration which used a hardware-based system as the references. Basically, NIRS and PET are the imaging modalities that give functional information, especially for brain activity.

Near infra-red spectroscopy is the optical imaging system that used near infra-red light to assess the functional state during brain activities. It can detect changes in the concentration of oxygenated and deoxygenated hemoglobin in blood [3]. The change in hemodynamic response is a good marker for assessing neural activity since the oxygenated level is dependent on brain activities and mostly NIRS measurement is done based on mental work in human [4]. NIRS measured the oxygenated level of blood, which similar to blood-oxygen-level dependent (BOLD) technique in fMRI. Nevertheless, the beauty of NIRS is it can detect the oxygenated changes while the subject is doing an activity. In view of the fact that NIRS is task related, there are many studies done involves NIRS measurement while the subject is doing a task such as an exercise using muscle [5], [6], playing a video game system [7], cycling [8] and verbal fluency task [9], [10].

PET is nuclear imaging technique that used radioactive to image the functional state inside the human body. It is involving the injection of a radiotracer into human body where the tracer is a biological compound of interest labelled with a positron emitters isotope such as ${ }^{11} \mathrm{C},{ }^{18} \mathrm{~F}$ and ${ }^{15} \mathrm{O}$ [11]. Specific tracers are chosen to illustrate the particular brain functions, for example, ${ }^{18} \mathrm{~F}$-2-deoxyglucose (18FDG) is used to investigate cerebral glucose metabolism, whereas $\mathrm{H}_{2}{ }^{15} \mathrm{O}$ is used to examine cerebral blood flow [11].

Both techniques NIRS and PET, have their own advantages and disadvantages. NIRS has high temporal resolution while PET image has a better spatial resolution [12]. NIRS also is relatively non-invasive technique, inexpensive compared to other modalities, safe in term of radioactive usage and portable. NIRS is completely silent, providing a nonintrusive environment and allowing for an easy presentation of auditory stimuli [13]. However, the depth penetration of light in NIRS is very low, therefore NIRS only measures hemoglobin concentration on the cortex region rather than in deeper brain structures [13], [14]. Thus, brain structures that lie deeper in the cortex or below it is not visible using NIRS techniques [13].

Therefore, by registering the NIRS data on PET image, it can optimize the advantages of NIRS and PET as well as improve the specificity of the information given by both methods. This paper describes how the process of registration of NIRS on PET image using hardware base registration.

\section{Materials and Methods}

\subsection{Study Design}

This study was done at Cyclotron and Radioisotope Center (CYRIC) Tohoku University. Fig. 1 illustrates the process to register the NIRS on the PET image. PET image is used as the reference image while NIRS data as the target image and the registration is based on a hardware-based system which is done using Polaris. NIRS data is relatively the probes location on the NIRS cap. Each subject undergoes NIRS examination and PET scanned separately and therefore Polaris markers were attached on NIRS cap as in Fig. 2a and subject wore the cap for both procedures. To register the NIRS on PET image, we transformed the NIRS probe location into the PET coordinate based on the Polaris marker location. 


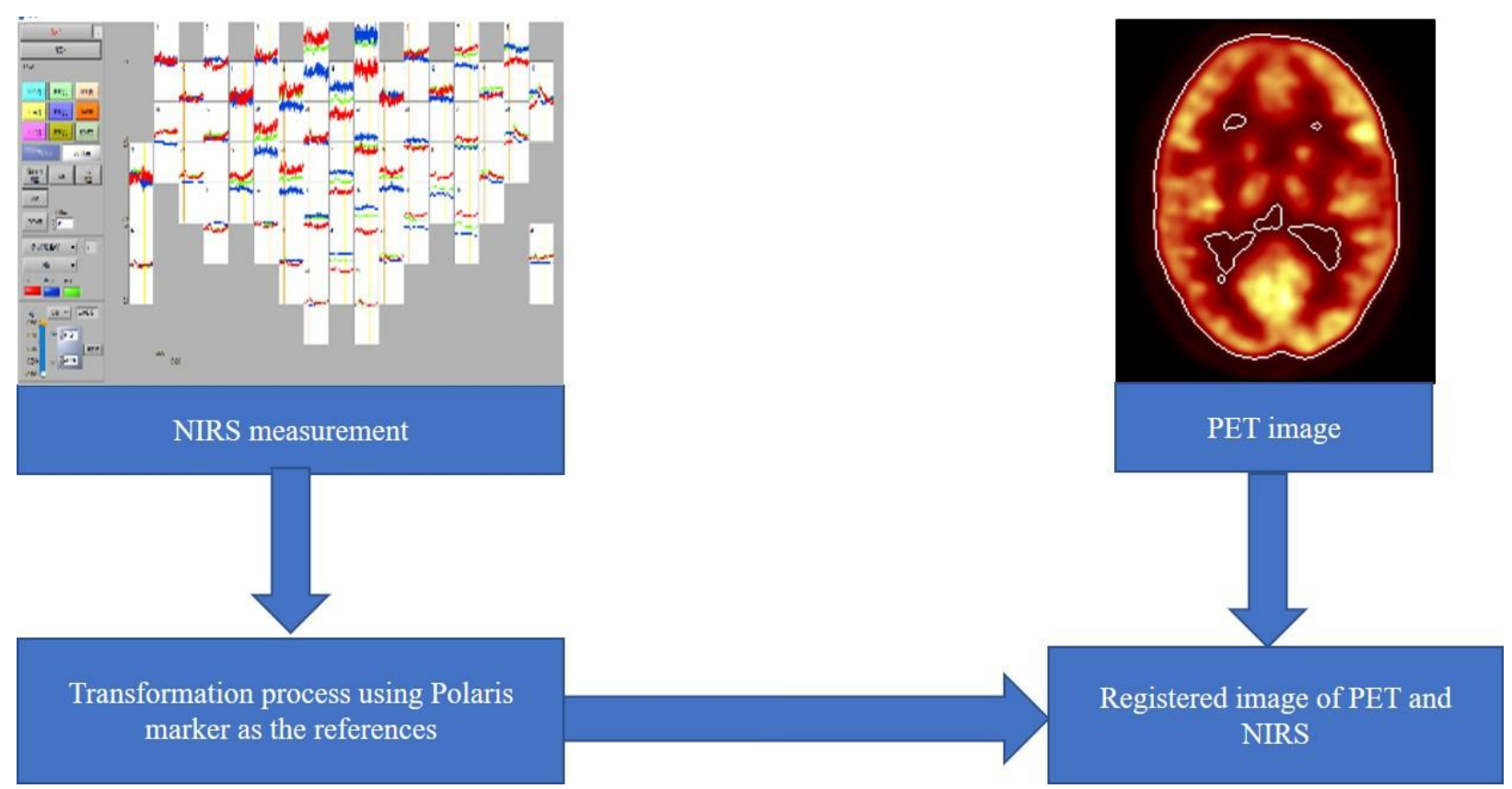

Fig. 1. The process to register the NIRS on PET image using Polaris marker as the reference. The NIRS measurement is undergoing transformation process beforehand register on PET image.

\subsection{Participants}

A total of eleven subjects participated in this study. All subject is male and healthy subject (age 20 to 25 years old). Subject with brain abnormalities was excluded from this study. Written consent was obtained from each subject before undergoing this experiment.

\subsection{Functional Near Infra-Red Spectroscopy}

During the NIRS task, the changes of hemodynamic response in the prefrontal cortex were recorded by NIRS FOREI 3000 system (Shimadzu Inc., Kyoto Japan). This NIRS system consists of transmitting probe which emits infrared light at three different wavelengths $(780,805$ and $830 \mathrm{~nm})$ and receiving probe which record the reflected infrared light. The probe is mounted on the NIRS cap and separate $3 \mathrm{~cm}$ apart each. The NIRS cap was fixed on subject head over the prefrontal regions and the location of NIRS probe was determined using FASTRAK 3D magnetizer.

\subsection{Positron Emission Tomography (PET)}

PET scanner used in this study was Eminence STARGATE PET-CT scanner (Shimadzu Inc., Kyoto, Japan). As this study only focuses on PET, we only used PET scanner instead of CT function. The scanning covered only brain part, which is about 5 minutes transmission scan for tissue attenuation correction and 20 minutes emission scan.

\subsection{Optical Tracking System}

The optical system used in this study was Polaris Vicra NDI as shown in Fig. 2b. Polaris use near infra-red to detect the 3D location of markers. It has two digital cameras that can detect location and orientation of multiple markers simultaneously. Polaris marker is made of the material that can reflect the infra-red light so that Polaris can detect the reflected light. Fig. 2a shows the sticker type marker while Fig. 2c shows the ball type Polaris marker. In this study, we used sticker type marker to attach on the NIRS cap while ball type marker was attached to the PET gantry. 


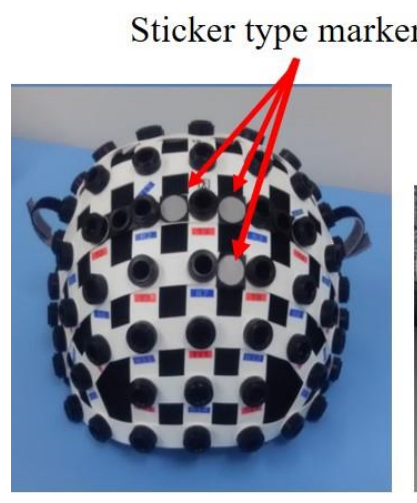

a

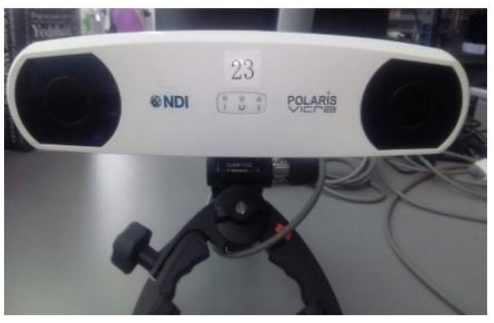

$\mathrm{b}$

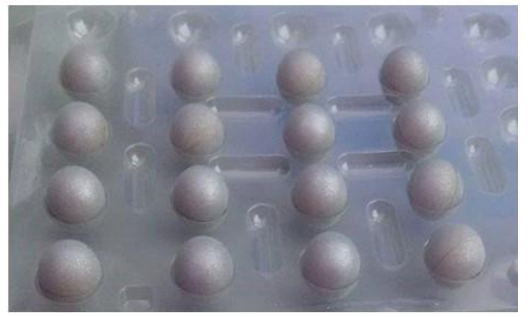

c

Fig. 2. a) The sticker type Polaris marker that attached to the subject cap b) The Polaris Vicra NDI and c) The ball type Polaris marker that used on the gantry.

\subsection{Measurement Procedures}

The whole procedure is shown in Fig. 3. The details of this study are found in elsewhere [15]. Before the preparation for the NIRS examination, the subject was inserted with normal saline drip into the left antecubital vein. Then the subject was asked to sit comfortably on the chair facing the PC monitor during the fixation of the NIRS cap. Three Polaris markers were attached to the NIRS cap as in Fig. 3 and subject wore the cap for both procedures. Since the NIRS and PET are done separately, the cap was fixed tightly on the subject head to prevent movement during the transition PET room later. Before fixing the NIRS probe, the location of all NIRS probes was determined using FASTRAK 3D magnetizer. After fixing all the NIRS probe on the cap, FDG-18 was injected into the subject through the left antecubital vein. The NIRS task started shortly after the injection and subject completed the task within 30 minutes as well as NIRS measurement. After the task finished, all the NIRS probes were removed from the cap and the subject was transferred to the PET room with the cap still on the subject head. Then the subject was laid out on the PET bed and the head is fixed to immobilize during the scanning. The setup is shown in Fig. 4. At the same time, Polaris is placed in between the PET gantry and CT gantry as shown in Fig. 4a. Polaris is connected to a personal computer and read the position of Polaris marker on the cap as well as the gantry marker during the PET scanned (Fig. 4b).

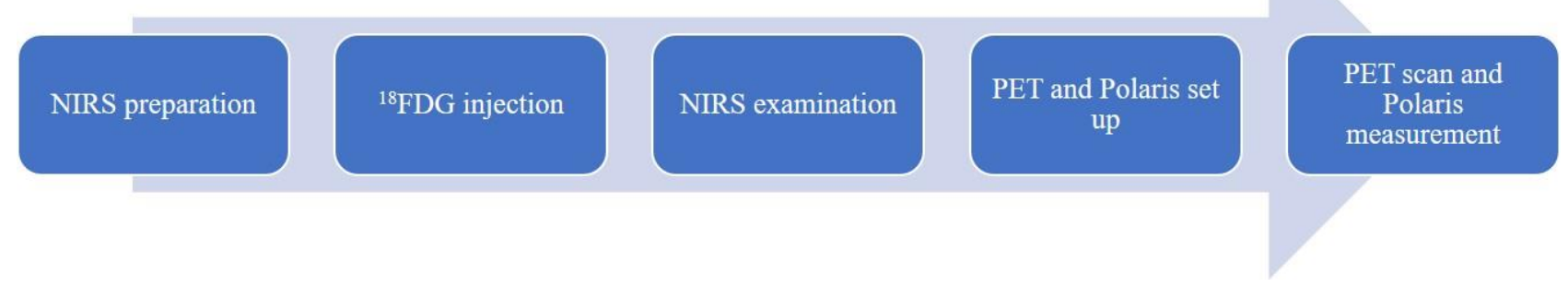

Fig. 3. The flow of the procedure of the measurement.

\subsection{The Registration}

The registration process started with the transformation of NIRS probe and Polaris marker to the PET coordinate. The transformation process is illustrated in Fig. 5. Considered we have three coordinate systems: 
1) NIRS; $C_{\text {NIRS }}$ 2) Polaris system; $C_{P O L}$ and 3) PET image; $C_{\text {PET. }}$ From the measurement done, we have coordinate of the probe, $\mathrm{P}_{\text {NIRS }}(\mathrm{x}, \mathrm{y}, \mathrm{z})$ in $\mathrm{C}_{\text {NIRS }}$ given by magnetic probe and the coordinate of marker $\mathrm{M}_{\mathrm{POL}}(\mathrm{x}$, $\mathrm{y}, \mathrm{z}$ ) in $\mathrm{C}_{\mathrm{POL}}$ given by Polaris. The transformation is done to convert these coordinate into $\mathrm{C}_{\mathrm{PET}}$. The transformation is divided into two steps, first is the conversion of $\mathrm{P}_{\mathrm{NIRS}}(\mathrm{x}, \mathrm{y}, \mathrm{z})$ to the $\mathrm{C}_{\mathrm{PET}}$ and second is the conversion of $\mathrm{M}_{\mathrm{POL}}(\mathrm{x}, \mathrm{y}, \mathrm{z})$ into $\mathrm{C}_{\mathrm{PET}}$. The $\mathrm{P}_{\mathrm{NIRS}}(\mathrm{x}, \mathrm{y}, \mathrm{z})$ was transformed to the $\mathrm{P}_{\mathrm{POL}}(\mathrm{x}, \mathrm{y}, \mathrm{z})$ in $\mathrm{C}_{\mathrm{POL}}$ using transformation matrix; $\mathrm{T}_{\mathrm{NIRS}}$ - POL as the beforehand transformed to $\mathrm{P}_{\mathrm{PET}}(\mathrm{x}, \mathrm{y}, \mathrm{z})$ in $\mathrm{C}_{\mathrm{PET}}$ using transformation matrix; $\mathrm{T}_{\mathrm{POL}}{ }^{\mathrm{PET}}$ (Fig. 5). The marker $\mathrm{M}_{\mathrm{POL}}(\mathrm{x}, \mathrm{y}, \mathrm{z})$ in $\mathrm{C}_{\mathrm{POL}}$ was transformed to $\mathrm{M}_{\mathrm{PET}}(\mathrm{x}, \mathrm{y}, \mathrm{z})$ in $\mathrm{C}_{\mathrm{PET}}$ using transformation matrix; $\mathrm{T}_{\mathrm{POL}} \rightarrow{ }^{\mathrm{PET}}$ (Fig. 5). The details of these transformation are given in the appendix. Thereafter, registration of the NIRS probe, as well as Polaris marker on the PET image was done. The transformation process gave the NIRS probe position in PET coordinate, СРЕT. By using in-house software, we did register the NIRS coordinate and Polaris marker on the PET image.
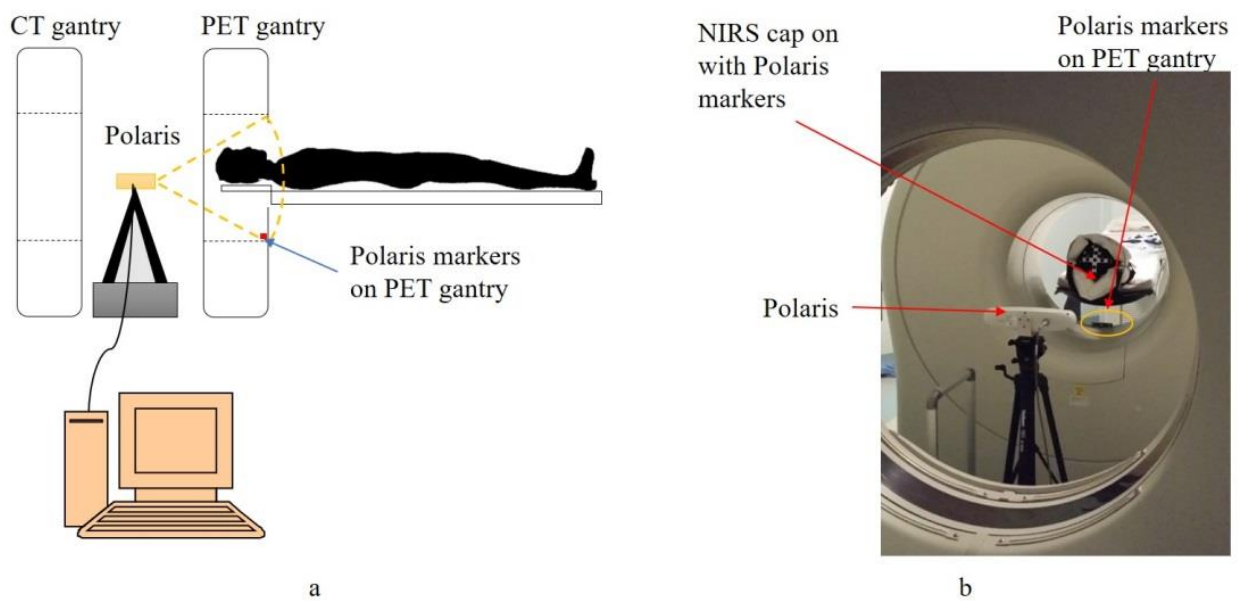

Fig. 4. a) Illustration of the Polaris and subject set up. The Polaris placed in between CT and PET gantry and connected to a personal computer system for the data recording. b) The position of the subject head as well as Polaris. Three Polaris markers are attached to the cap and three markers on PET gantry.

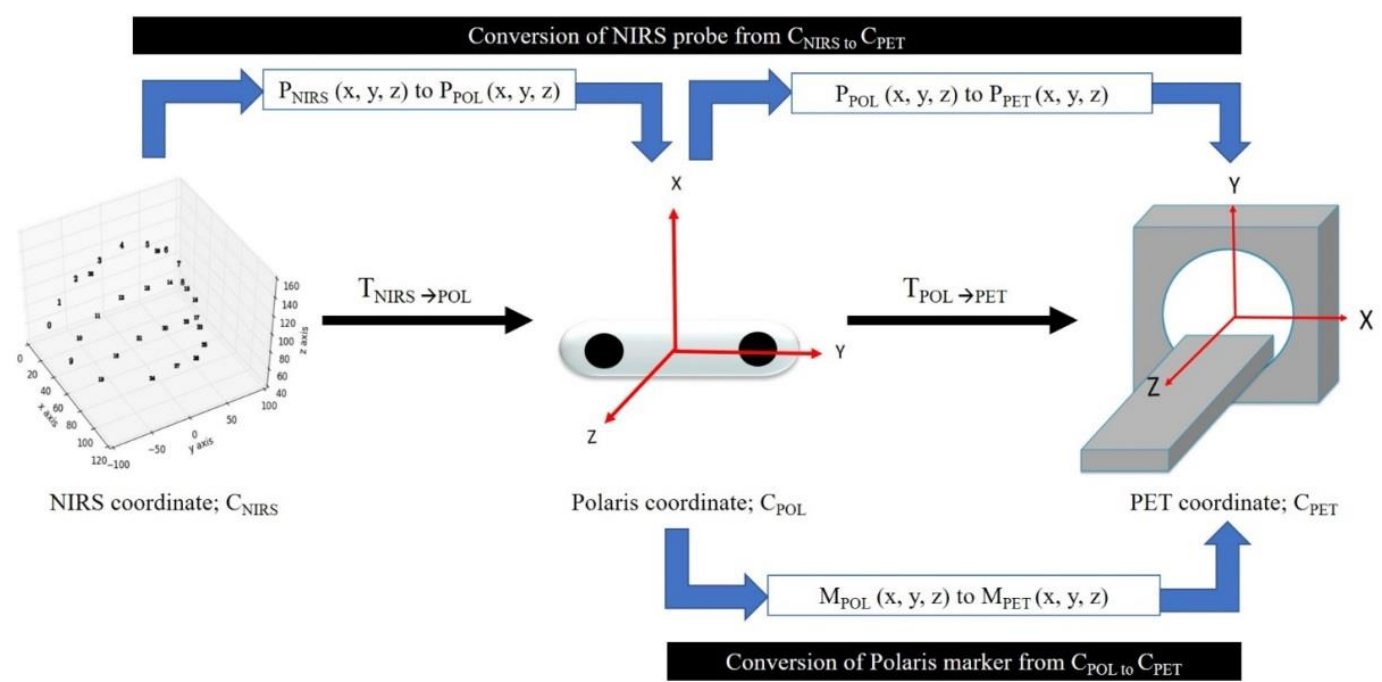

Fig. 5. The entire flow of transformation process. The 30 probes were transformed to Polaris coordinate beforehand transformed to $\mathrm{C}_{\mathrm{PET}}$, while the Polaris markers were transformed directly to $\mathrm{C}_{\mathrm{PET}}$.

\section{Result}


The transformation of the probe from $\mathrm{C}_{\mathrm{NIRS}}$ to $\mathrm{C}_{\mathrm{POL}}$ done for all eleven subjects by using Kabsh algorithm

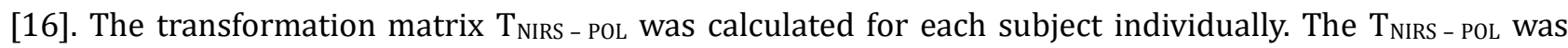
calculated form conversion of $\mathrm{M}_{\mathrm{NIRS}}$ to $\mathrm{M}_{\mathrm{POL}}$ and error of each subject was determined. Seven subjects out of eleven subjects give error less than $1 \mathrm{~mm}$, while another four subjects give error below than $5 \mathrm{~mm}$. The error was calculated by comparing the estimated $\mathrm{M}_{\mathrm{POL}}$ coordinate using calculated $\mathrm{T}_{\mathrm{NIRS} \text { - POL }}$ and the actual value $\mathrm{M}_{\mathrm{POL}}$ coordinate. For the transformation from $\mathrm{C}_{\mathrm{POL}}$ to $\mathrm{C}_{\mathrm{PET}}$, the transformation matrix was determined by transformed the ${ }^{22} \mathrm{Na}$ position given by Polaris to the known position of ${ }^{22} \mathrm{Na}$ on PET image. Therefore, for all subject, the transformation from Polaris to $\mathrm{C}_{\mathrm{PET}}$ were based on only one value of $\mathrm{T}_{\mathrm{POL}} \rightarrow{ }^{\mathrm{PET}}$. Fig. 6 shows the activation area based on NIRS task which is view on PET image. The NIRS task consists of three tasks, therefore, we can view the activation area on PET image for the task 1, task 2 and task 3 such in Fig. 6a, 6b and $6 \mathrm{c}$.

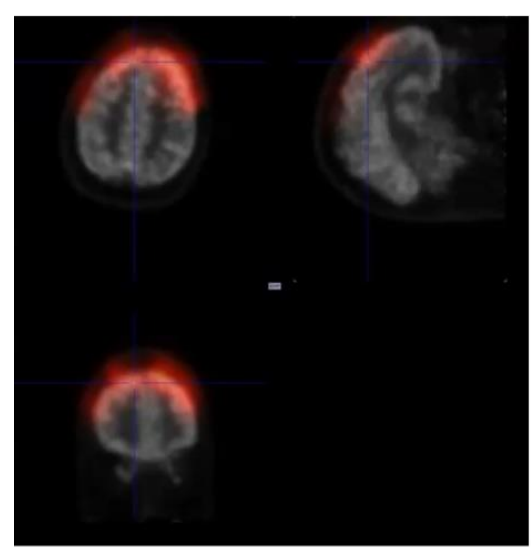

a

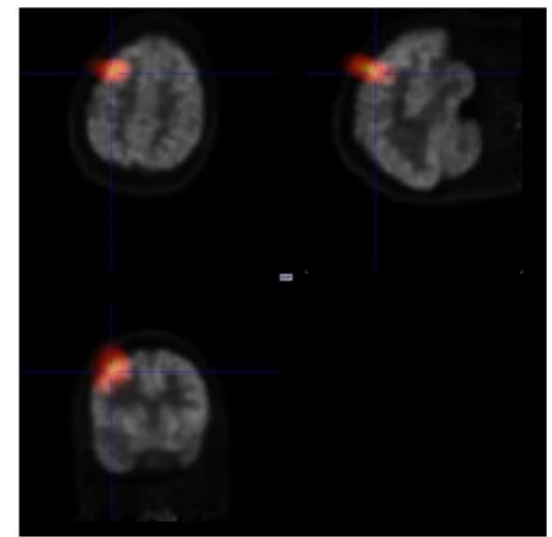

b

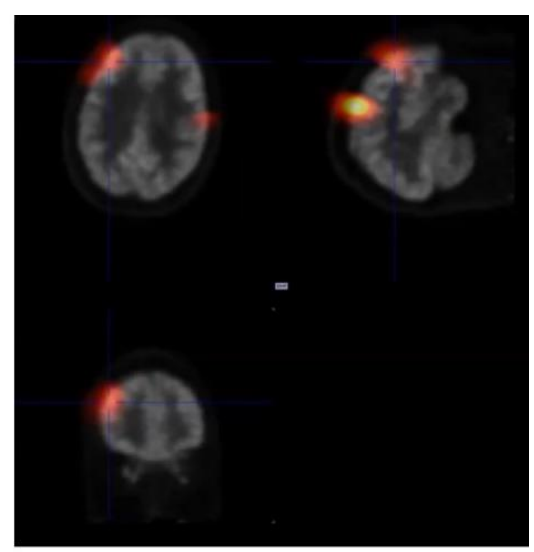

C

Fig. 6. The activation area based on NIRS task which observed on PET image. The red colour shows the activation area during NIRS task. a) The activation area during the first task, b) The activation area during the second task and 3) The activation area during the third task.

\section{Discussion}

We demonstrated the registration of NIRS on PET image for all eleven subjects using geometrical transformation methods. The aim of this study is to register the NIRS image on PET image, therefore the result is focused on the translation of the NIRS probe coordinate and Polaris marker coordinate to the PET coordinate. The registration of NIRS on PET image is considered successful and we can view the NIRS activation area on the PET image. The NIRS measurement depends on the length of the light travel, thus NIRS result is mostly in the cortex region of the brain. Thus, by transformed the NIRS probe to the brain surface of PET image, we can see clearly where the changes happened during the task performed. As the task in this study is the cognitive task, therefore the response is in the frontal area as shown in Fig. 6.

Our approach is to bring the NIRS on PET together to optimize the information given by NIRS and PET alone. NIRS alone can detect the change of oxyhemoglobin and deoxyhemoglobin in blood during brain activity. The activation of the brain related to the changes of blood flow to the active area and changes of oxyhemoglobin and deoxyhemoglobin in blood [4]. Therefore, NIRS is very beneficial when measures during brain activity. Several NIRS studies in demonstrated that changes in brain activity can be assessed non-invasively [17]. There are several types of brain activity have been assessed during motor activity, visual activation, auditory stimulation and performance of cognitive task [8], [17]. Meanwhile, 18FDG-PET is used to investigate cerebral glucose metabolism wherein certain activated brain regions, the demand for 
glucose and oxygen may increase due to the increased regional brain energy metabolism. Therefore, performed PET scan after the NIRS task could visualize the active area where high glucose uptake due to the NIRS task. Despite the fact that NIRS has a critical issue on spatial resolution, but then it gives a good temporal resolution. On the other hand, PET produces better spatial resolution compare to NIRS. Therefore, this work could give better consequence where we can view the activation area during NIRS task on PET image.

In this study, the registration technique is done base on extrinsic method [18]. We use Polaris marker as the artificial object attached on the subject and this technique also is hardware base registration where we used Polaris as the optical tracking system to register both images. However, there is an error on the transformation matrix from NIRS to PET for a certain subject. This error is probably due to the movement of the cap during the transfer from NIRS room to PET room and this cause rotation of the marker on the cap although we already fix the cap tightly to reduce the movement.

Our technique is the initial phase to produce a better fusion between NIRS and PET as the registration is the first step of image fusion [2]. Recently, there are many approaches of multimodal imaging such as PET-CT, MRI-CT and PET-MRI [2], [19], [20]. Generally, all these multimodal images have more advantage as it gives information anatomical and physiological and more clinical benefits [1]. However, PET-CT technique involves large radiation dose as the patient receive radiation from radiotracer injected during PET as well as $\mathrm{x}$-ray from CT [21]. For the PET-MRI or CT-MRI, those technique is costly compared to NIRS which is considerably lower. Thus, for those who already have PET machine, the installation of NIRS will be less expensive compared to installing the CT or MRI.

Another technique similar to our study is PET-fMRI as the BOLD technique of fMRI also monitor the oxygen level in blood. However, NIRS can detect the change of oxyhemoglobin, deoxyhemoglobin as well as total hemoglobin in blood while the BOLD response in fMRI only is related to deoxyhemoglobin only [13]. Another advantage is NIRS is task related, so that we can visualize the hemodynamic level change during task and NIRS is completely silent, provide a nonintrusive environment and allowing for an easy presentation of auditory stimuli [13].

Recent work on NIRS with combination other imaging technique are done simultaneously. NIRS is a freely moving tool, therefore the combination of NIRS-PET or NIRS-fMRI done simultaneously where the NIRS tools are brought to the bedside and subject performed the task during both measurements [22]-[24]. Although NIRS is portable and can bring along to the bedside in PET or MRI room, the task is limited due to space and a complicated setup is required for simultaneous measurement. These will lead to inconvenience during the task done and probably contribute the motion in PET. In our approach, the NIRS and PET are done separately, give more space and comfortable environment during the NIRS task and then go for PET scan with less motion during the scanning.

This work has the limitation as well. As the PET and NIRS are done separately, the subject was required to wear the NIRS cap for a longer time such in this study for one hour. Although the cap is soft, it may bring uncomfortable for certain subject especially elderly or child. Besides that, the registration may take time as the transformation is done for each subject. In future, a standard software should be developed for the effectiveness of the registration process.

\section{Conclusion}

This registration of NIRS on the PET image is considered successful but then again, this registration technique should be tested on another object such as phantom to validate this registration method. This study is relatively new, but then it is a respectable jump to make a better fusion image of NIRS and PET. The main challenge of NIRS-PET fusion is the need to visualize deeper in the brain structure since brain 
activities not only in the cortex region. In future, clinical aspect should be considered in order to gain as much clinical information as well as provide a better diagnosis and to expand the patient benefits.

\section{Appendix}

The transformation of the NIRS cap, $\mathrm{P}_{\mathrm{NIRS}}(\mathrm{x}, \mathrm{y}, \mathrm{z})$ and Polaris marker; $\mathrm{M}_{\mathrm{POL}}(\mathrm{x}, \mathrm{y}, \mathrm{z})$ to the $\mathrm{C}_{\mathrm{PET}}$.

1) Conversion of $P_{\text {NIRS }}(x, y, z)$ into $C_{P O L}$

Based on probe coordinate given by magnetic probe, we calculated the coordinate of the marker in the $\mathrm{C}_{\mathrm{NIRS}}$. The marker location, $\mathrm{M}_{\mathrm{NIRS}}(\mathrm{x}, \mathrm{y}, \mathrm{z})$ in $\mathrm{C}_{\mathrm{NIRS}}$ is corresponded to the marker location, $\mathrm{M}_{\mathrm{POL}}(\mathrm{x}, \mathrm{y}, \mathrm{z})$ in $\mathrm{C}_{\mathrm{POL}}$, as shows in the following relationship;

$$
\mathrm{M}_{\mathrm{POL}}=\mathrm{T}_{\mathrm{NIRS}} \rightarrow^{\mathrm{POL}}{ }^{*} \mathrm{M}_{\mathrm{NIRS}}
$$

Therefore, $\mathrm{T}_{\mathrm{NIRS}} \rightarrow{ }^{\mathrm{POL}}$ is given by the following relationship;

$$
\mathrm{T}_{\mathrm{NIRS}} \rightarrow{ }^{\mathrm{POL}}=\mathrm{M}_{\mathrm{POL}}{ }^{*} \mathrm{M}_{\mathrm{NIRS}}{ }^{-1}
$$

By using $\mathrm{T}_{\mathrm{NIRS}} \rightarrow{ }^{\mathrm{POL}}$, coordinate of probe, $\mathrm{P}_{\mathrm{POL}}(\mathrm{x}, \mathrm{y}, \mathrm{z})$ in $\mathrm{C}_{\mathrm{POL}}$ was calculated by the following equation;

$$
\mathrm{P}_{\mathrm{POL}}=\mathrm{T}_{\mathrm{NIRS}} \rightarrow{ }^{\mathrm{POL}}{ }^{*} \mathrm{P}_{\mathrm{NIRS}}
$$

2) Conversion of $P_{P O L}(x, y, z)$ and $M_{P O L}(x, y, z)$ into $C_{P E T}$

The coordinate of probe, $P_{\mathrm{POL}}(\mathrm{x}, \mathrm{y}, \mathrm{z})$ and marker, $\mathrm{M}_{\mathrm{POL}}(\mathrm{x}, \mathrm{y}, \mathrm{z})$ in $\mathrm{C}_{\mathrm{POL}}$ is corresponded to $\mathrm{P}_{\mathrm{PET}}(\mathrm{x}, \mathrm{y}, \mathrm{z})$ and $\mathrm{M}_{\mathrm{PET}}(\mathrm{x}, \mathrm{y}, \mathrm{z})$ in $\mathrm{C}_{\mathrm{PET}}$, as given by the following relationship;

$$
\mathrm{P}_{\mathrm{PET}}=\mathrm{T}_{\mathrm{POL}} \rightarrow{ }^{\mathrm{PET}} * \mathrm{P}_{\mathrm{POL}} \text { and } \mathrm{M}_{\mathrm{PET}}=\mathrm{T}_{\mathrm{POL}} \rightarrow{ }^{\mathrm{PET}} * \mathrm{M}_{\mathrm{POL}}
$$

To calculate the $\mathrm{T}_{\mathrm{POL}} \rightarrow$ PET, we did an experiment using sodium point source $\left({ }^{22} \mathrm{Na}\right)$ to get the position of Polaris marker in PET coordinate as well as Polaris coordinate. This experiment was setup same as in PET scanning and Polaris measurement setup except that ${ }^{22} \mathrm{Na}$ source was put on the PET couch. The ${ }^{22 \mathrm{Na}}$ source was surrounded by Polaris markers so that we get the source position in $\mathrm{C}_{\mathrm{POL}}$ as well as in $\mathrm{C}_{\mathrm{PET}}$. Thus, the coordinate of ${ }^{22} \mathrm{NaPOL}(\mathrm{x}, \mathrm{y}, \mathrm{z})$ in $\mathrm{C}_{\mathrm{POL}}$ is corresponded to ${ }^{22} \mathrm{Na} \mathrm{P}_{\mathrm{PET}}(\mathrm{x}, \mathrm{y}, \mathrm{z})$ in $\mathrm{C}_{\mathrm{PET}}$ as in the following relationship;

$$
{ }^{22} \mathrm{Na} \mathrm{PET}_{\mathrm{PET}}=\mathrm{T}_{\mathrm{POL}}{ }_{\mathrm{PET}} * 22 \mathrm{Na} \mathrm{POL}
$$

Therefore, $\mathrm{T}_{\mathrm{POL}} \rightarrow{ }^{\mathrm{PET}}$ is given by the following relationship;

$$
\mathrm{T}_{\mathrm{POL} \rightarrow \mathrm{PET}}={ }^{22} \mathrm{Na} \mathrm{PET} * 22 \mathrm{Na}_{\mathrm{POL}^{-1}}
$$

By using $\mathrm{T}_{\mathrm{POL}} \rightarrow{ }^{\mathrm{PET}}$, coordinate of probe, $\mathrm{PPET}(\mathrm{x}, \mathrm{y}, \mathrm{z})$ in $\mathrm{C}_{\mathrm{PET}}$ was calculated by the following equation; $\mathrm{P}_{\mathrm{PET}}=\mathrm{T}_{\mathrm{POL}} \rightarrow{ }^{\mathrm{PET}} * \mathrm{P}_{\mathrm{POL}}$ as well as coordinate of marker coordinate; $\mathrm{M}_{\mathrm{PET}}=\mathrm{T}_{\mathrm{POL}} \rightarrow{ }^{\mathrm{PET}} * \mathrm{M}_{\mathrm{POL}}$

\section{Acknowledgment}

This study was supported by Grants-in-Aid for Scientific Research No. 17H04118 from the Ministry of Education, Culture, Sports, Science and Technology (MEXT), Japanese Government and partly supported by a collaboration research grant from GlaxoSmithKline (to M. Tashiro).

\section{Conflict of Interest}

H. Watabe and M. Tashiro have potential conflicts of interest regarding the grant from GlaxoSmithKline.

\section{References}

[1] Fitzpatrick, J. M., Hill, D. L. G., \& Maurer, C. R. (2004). Image registration. Handbook of Medical Imaging 447-514. SPIE Press.

[2] El-Zahraa, F., El-Gamal, A., Elmogy, M., \& Atwan, A. (2016). Current trends in medical image registration and fusion. Egypt Informatics Journal, 17, 99-124.

[3] León-carrión, J., \& León-domínguez, U. (2012). Functional near-infrared spectroscopy (fNIRS): Principles and neuroscientific applications. Neuroimaging - Methods, 47-74. 
[4] Hoshi, Y., \& Tamura, M. (1993). Detection of dynamic changes in cerebral oxygenation coupled to neuronal function during mental work in man. Neuroscience Letters, 150, 5-8.

[5] Nielsen, H. B., Boesen, M., \& Secher, N. H. (2001). Near-infrared spectroscopy determined brain and muscle oxygenation during exercise with normal and resistive breathing. Acta Physiol Scand, 171, 63-70.

[6] Perrey, S. (2008). Non-invasive NIR spectroscopy of human brain function during exercise. Methods, 45(4), 289-299.

[7] Karim, H., Schmidt, B., Dart, D., Beluk, N., \& Huppert, T. (2012). Functional near-infrared spectroscopy (fNIRS) of brain function during active balancing using a video game system. Gait and Posture, 35(3), 367-372.

[8] Piper, S. K., Krueger, A., Koch, S. P., Mehnert, J., Habermehl, C., \& Steinbrink, J., et al. (2014). A wearable multi-channel fNIRS system for brain imaging in freely moving subjects. Neuroimage, 85, 64-71.

[9] Takahashi, T., Takikawa, Y., Kawagoe, R., Shibuya, S., Iwano, T., \& Kitazawa, S. (2011). Influence of skin blood flow on near-infrared spectroscopy signals measured on the forehead during a verbal fluency task. Neuroimage, 57(3), 991-1002.

[10] Arai, H., Takano, M., Miyakawa, K., Ota, T., Takahashi, T., \& Asaka, H., et al. (2006). A quantitative near-infrared spectroscopy study: A decrease in cerebral hemoglobin oxygenation in Alzheimer's disease and mild cognitive impairment. Brain and Cognition, 61(2),189-194.

[11] Tai, Y., \& Piccini, P. (2004). Applications of positron emission tomography (PET) in neurology. Journal of Neurology, Neurosurgery, and Psychiatry, 75(5), 669-676.

[12] Chou, P. H., \& Lan, T. H. (2013). The role of near-infrared spectroscopy in Alzheimer's disease. Journal of Clinical Gerontology and Geriatrics, 4(2), 33-6.

[13] Gervain, J. (2015). Near-infrared spectroscopy. International Encyclopedia of the Social \& Behavioral Sciences, 2(16), 387-396.

[14] Huppert, T. J., Diamond, S. G., Franceschini, M. A., \& Boas D. A. (2009). HomER: A review of time-series analysis methods for near-infrared spectroscopy of the brain. Applied Optics, 48(10), D280-D298.

[15] Kikuchi, A., Fairuz, B. M. N, Inami, A., Attayeb, M., Watanuki, S., \& Miyake, M., et al. (2018). Effects of levocetirizine and diphenhydramine on regional glucose metabolic changes and hemodynamic responses in the human prefrontal cortex during cognitive tasks. Human Psychopharmacology: Clinical and Experimental.

[16] Wolfgang, K. (1976). A solution for the best rotation to related two sets of vectors. Acta Crystallographica Section A, (A32), 922-923

[17] Villringer, A., \& Chance, B. (1997). Non-invasive optical spectroscopy and imaging of human brain function. Trends in Neurosciences, 20(10), 435-442.

[18] Maintz, J. B. A., \& Viergever, M. A. (1996). An overview of medical image registration methods. Nature, 12(6), 1-22.

[19] Gong, S. J., Graeme, J., O'Keefe, \& Scott, A. M. (2005). Comparison and evaluation of PET/CT image registration. Proceedings of the 2005 IEEE Engineering in Medicine and Biology 27th Annual Conference (pp. 1599-603).

[20] Alexander, W. S., Hans, F. W., Armin, Kold., Martin, S. J., \& Bernd, J. P. (2010). Combined PET/MRI: One step further in multimodality imaging. Trends in Molecular Medicine, 16(11), 508-15.

[21] Akin, E. A., \& George, F. (2012). Considerations Regarding Radiation Exposure in Performing FDG-PET-CT, $1-7$.

[22] Hock, C., Villringer, K., Müller-Spahn, F., Wenzel, R., Heekeren, H., \& Schuh-Hofer, S., et al. (1997). Decrease in parietal cerebral hemoglobin oxygenation during performance of a verbal fluency task in 
patients with Alzheimer's disease monitored by means of near-infrared spectroscopy (NIRS) Correlation with simultaneous rCBF-PET measurements. Brain Research, 755(2), 293-303.

[23] Tak, S., Yoon, S. J., Jang, J., Yoo, K., Jeong, Y., \& Ye, J. C. (2011). Quantitative analysis of hemodynamic and metabolic changes in subcortical vascular dementia using simultaneous near-infrared spectroscopy and fMRI measurements. NeuroImage, 55(1), 176-84.

[24] Steinbrink, J., Villringer, A., Kempf, F., Haux, D., Boden, S., \& Obrig, H. (2006). Illuminating the BOLD signal: combined fMRI-fNIRS studies. Magnetic Resonance Imaging, 24(4), 495-505.

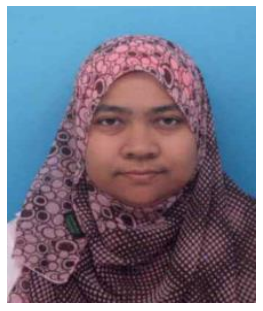

Fairuz binti Mohd Nasir was born in Kedah, Malaysia. She graduated with the MSc in medical physics and the BSc in medical radiation from University Sains Malaysia. She works as lecturer at Faculty of Health Science, in University Sultan Zainal Abidin, Malaysia from 2010 till present. She holds a license of Radiation Protection Officer since 2007 and was appointed as Radiation Protection Officer since 2007 till 2015. Currently, she is a PhD student in the Graduate School of Biomedical Engineering, Tohoku University, Japan. Her current study is about registering the NIRS and PET. She has presented her works during Japanese Society of Nuclear Medicine 2016 conference in Nagoya, Japan. Her research interest is radiation physics, image processing, and radiation protection.

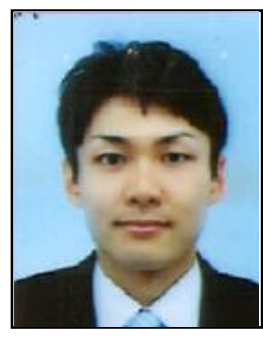

Asuka Kikuchi is a M.S. student in Graduate School of Medicine in the Tohoku University. He received B.S. degree in Health Science from Niigata University in 2016. His research fields are molecular imaging using Positron Emission Tomography (PET), Magnetic Resonance Imaging (MRI) and Near Infrared Spectroscopy (NIRS).

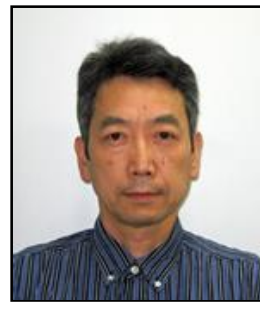

Shoichi Watanuki was born in Sapporo, Japan in 1957. He graduated from the Department of Radiologic Technology, School of Allied Medical Sciences, Hirosaki University, obtaining a radiologic technologist license. He was a radiologic technologist at Tenshi Hospital from 1980 to 1981 and at Japan Environment Research Co. from 1981 to 1983. He was a technical official at Cyclotron and Radioisotope Center (CYRIC), Tohoku University from 1983 to 2005, and he has been a research assistant since 2005. His current research interests are quality control of positron emission tomography system and its image data.

Mr. Watanuki is a member of the Japanese Society of Nuclear Medicine and the Japanese Society of Nuclear Medicine Technology.

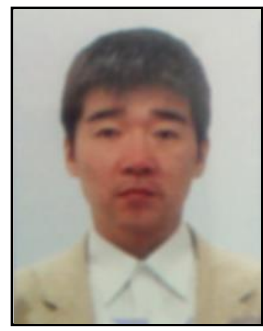

Masayasu Miayke is a research associate in Cyclotron and Radioisotope Center, Tohoku University, Japan from 2004, got his master's degree in engineering from Tohoku University in 2004.

He is interested in radiation detectors for PET camera, developed a prototype of a positron emission mammography camera. He is interested in computer networks, constructs network systems for research and education. 


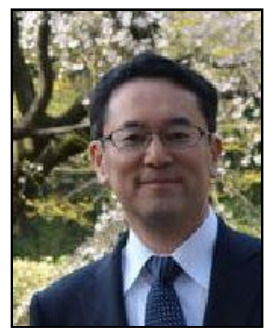

Manabu Tashiro was born in Nagano, Japan, in 1966. He received a M.D. degree from Shinshu University School of Medicine and was licensed as a medical doctor in 1994. He worked as a physician from 1994 to 1996. He received Ph.D. degree for medical sciences from Tohoku University Graduate School of Medicine, Japan, in 2000. He also studied as a guest physician/researcher at Division of Nuclear Medicine, Freiburg University Hospital, Germany from 1998-2001. He was an assistant professor in the Department of Pharmacology of Graduate School of Medicine of Tohoku University from 2001 to 2005, and a lecturer from 2005 to 2007 and an associate professor from 2007 to 2012 at the Division of Cyclotron Nuclear Medicine, Cyclotron and Radioisotope Center, Tohoku University. He is currently a professor and a vice-director of Cyclotron and Radioisotope Center, Tohoku University, Japan. His current research interests include functional/molecular imaging and clinical pharmacology.

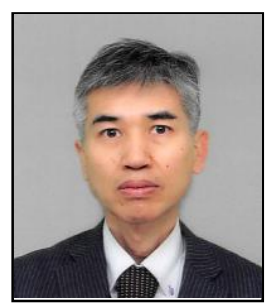

Hiroshi Watabe was born in Shizuoka, Japan in 1967. He was received Ph.D. in nuclear engineering from Tohoku University, Sendai, Japan in 1995. During his Ph.D. course, he was at Medical Research Council Cyclotron Unit, Hammersmith Hospital, London, UK in 1993 and 1994. In 1995-2009, he was at National Cardiovascular Center Research Institute, Osaka, Japan as a research staff and a section chief. In 1997-1999, he spent as a visiting fellow in PET Department, National Institutes of Health, Bethesda, U.S.A. From 2009 to 2013, he was an associate professor of Department of Molecular Imaging in Medicine, Osaka University Graduate School of Medicine, Osaka, Japan. Since 2013, he has been in Division of Radiation Protection \& Safety Control, Cyclotron and Radioisotope Center, Tohoku University, Sendai, Japan. Since 2015, he has been a professor. His research interest includes nuclear medicine physics, nuclear engineering in medicine and radiation dosimetry. 